\title{
Satellite Data Assimilation for Naval Undersea Capability Improvement
}

\author{
Peter C. Chu, chu@nps.navy.mil \\ Michael D. Perry, mdperry@nps.navy.mil \\ Naval Postgraduate School, Monterey, CA 93943 \\ Eric L. Gottshall, eric.gottshall@,navy.mil \\ SPAWAR, San Diego, CA 92110 \\ David S. Cwalina, Cwalinads@,npt.nuwc.navy.mil \\ Naval Undersea Warfare Center, Newport, RI 02841
}

\begin{abstract}
Impact of the satellite data assimilation on the naval undersea capability is investigated using the ocean hydrographic data without and with satellite data assimilation. The former is the Navy's Global Digital Environmental Model (GDEM) providing the monthly mean; and the latter is the Modular Ocean Data Assimilation System (MODAS) proving the synoptic data. The two environmental datasets are taken as the input into the Weapon Acoustic Preset Program to determine the suggested presets for a Mk 48 torpedo. The acoustic coverage area generated by the program will be used as the metric to compare the two sets of outputs. The output presets were created for two different scenarios, an ASUW and an ASW, and three different depth bands, shallow, mid, and deep. After analyzing the output, it became clear that there was a great difference in the presets for the shallow depth band, and that as depth increased, the difference between the presets decreased. Therefore, the MODAS data (in turn the satellite data assimilation) was optimized in the shallow depth band. The ASW presets also seemed to be slightly more resistant to differences in the presets than was the ASUW scenario.
\end{abstract}

Key Words - Satellite data, GDEM, MODAS, weapon acoustic preset

\section{INTRODUCTION}

Even with all the high technology weapons onboard U.S. Navy ships today, the difference between success and failure often comes down to our understanding and knowledge of the environment in which we are operating. Accurately predicting the ocean environment is a critical factor in using our detection systems to find a target and in setting our weapons to prosecute a target (Gottshall, 1997 [1]; Chu et al., 1998 [2]). From the ocean temperature and salinity, the sound velocity

\footnotetext{
${ }^{1}$ This work was sponsored by SPAWAR PMW 155-3.
}

profiles (SVP) can be calculated. SVPs are a key input used by U.S. Navy weapons programs to predict weapon performance in the medium. The trick lies in finding the degree to which the effectiveness of the weapon systems is tied to the accuracy of the ocean predictions.

The U.S. Navy's Meteorological and Oceanographic (METOC) community currently uses three different methods to obtain representative SVPs of the ocean: climatology, in-situ measurements, and data (including satellite data) assimilation. The climatological data provides the background SVP information that might not be current. The Generalized Digital Environmental Model (GDEM) is an example of a climatological system that provides long term mean temperature, salinity, and sound speed profiles. . The in-situ measurements such as the conductivity-temperaturedepth (CTD) and expendable bathythermographs (XBT) casts may give accurate and timely information, however, it is not likely to have large spatial and temporal coverage that U.S. ships are going to be operating. The data assimilation system is to use the climatology as the initial guess and then to obtain synoptic SVPs using satellite and in-situ data. The Modular Ocean Data Assimilation System (MODAS) utilizes sea surface height ( $\mathrm{SSH}$ ) and sea surface temperature (SST) in this way to make nowcasts of the ocean environment.

Value-aided satellite data assimilation on the undersea weapon systems can be evaluated using the SVP input data from MODAS (with satellite data assimilation) and GDEM (climatology without satellite data assimilation). The question also arises of how many altimeters are necessary to generate an optimal MODAS field. Too few inputs could result in an inaccurate MODAS field, which in turn leads to decreased weapon effectiveness. There must also be some point at which the addition of another altimeter is going to add a negligible increase in 
effectiveness. This superfluous altimeter is then simply a waste of money that could be spent on a useful system.

The purpose of this study is to quantify the advantage gained from the use of MODAS data rather than climatology. The study will specifically cover the benefits of MODAS data over climatology when using their respective SVP's to determine torpedo settings. These settings result in acoustic coverage percentages that will be used as the metric to compare the two types of data.

\section{NAVY'S METOC MODELS AND DATA}

\section{A. GDEM}

GDEM is a four dimensional (latitude, longitude, depth and time) digital model maintained by the Naval Oceanographic Office. GDEM was generated using over seven million temperature and salinity observations, most of them drawn from the Master Oceanographic Observation Data Set (MOODS). Globally GDEM has a resolution of $1 / 2^{\circ}$ degree. However, in a few select areas, higher resolutions are available. In order to represent the mean vertical distribution of temperature and salinity for grid squares, GDEM determines analytical curves to fit to the individual profiles (Teague et. al., 1990 [3])

Before curves can be fitted to the data, quality control must be implemented that removes anomalous features or bad observations. The data is checked for proper range and static stability, and it is checked to ensure that it has not been misplaced in location or season. Once the data has been inspected for quality, curves are fitted to the data. From the mathematical expressions that represent the curves, coefficients are determined. It is these coefficients that will be averaged. It can be shown that the coefficients resulting from averaged data are not the same as the averaged coefficients of the data. In order to minimize the number of coefficients necessary to generate smooth curves different families of curves are used for different depth ranges. This necessitates the careful selection of matching conditions in order to ensure that no discontinuities in the vertical gradients occur. Separate computation of temperature and salinity allow the results to be checked against each other to ensure stable densities.

\section{B. Modular Ocean Data Assimilation System}

MODAS is a collection of over 100 FORTRAN programs and UNIX scripts that can be combined to generate a number of different products. A few examples of MODAS programs include data sorting, data crossvalidation, data assimilation, and profile extension. This modularity allows MODAS to be quickly and easily modified to handle problems or new requirements as they arise. MODAS has varying degrees of resolution starting at $1 / 2^{\circ}$ in the open ocean increasing to $1 / 4^{\circ}$ in coastal seas and increasing again $1 / 8^{\circ}$ near the coast (Fox et al., 2002 [4]). To generate nowcasts and forecasts, the MODAS system uses a relocatable version of the Princeton Ocean Model (POM). To initialize the POM MODAS temperature and salinity grids, geostrophically estimated currents, or extracted currents from other POM's can be used (Fox, 2003 [4]).

One of the most important features of MODAS is its use of dynamic climatology. Dynamic climatology is the incorporation of additional information into the historical climatology in order to portray transient features that are not represented by the climatology. Two useful quantities that are easily gathered from satellites are sea surface height (SSH) and sea surface temperature (SST). While SST from altimeters can be used directly, the $\mathrm{SSH}$, which is measured as the total height relative to the proscribed mean, must be converted into a steric height anomaly in order to be used. 2D SST and SSH fields are generated from point observations through the use of optimal interpolation.

Optimal interpolation is a process by which the interpolated temperature or salinity anomaly is determined as the linear combination of the observed anomalies. Each of the anomalies is given a weight that accounts for variation in temporal and spatial sampling. Weights are computed by minimizing the least square difference between the interpolated value and the true value at the grid point and by solving the equations

$$
\sum_{j=1}^{N} \alpha_{j} \mu_{i j}+\lambda^{-2} \alpha_{i}=\mu_{G i}
$$

where $\alpha_{i}$ are the weights, $\lambda$ is the signal to noise ratio, $\mu_{i j}$ is the autocorrelation between locations $i$ and $j$, and $\mu_{G i}$ is the autocorrelation between the grid point and $i$. For each grid node location matrix inversion is used to solve the system of $N$ equations for the $\mathrm{N}$ unknown weights. The other parameters are computed using the first guess field, MOODS profiles, and climatology. Using this process any new observation can be interpolated into the appropriate MODAS grid node.

The first guess field, the prior days 2D SST field, or the weighted average of 35 days of altimeter data respectively, is subtracted from the new observations, and the resulting deviations are interpolated to produce a field of deviation. This is added to the first guess field to generate the new 2D field. For the first iteration of the optimal interpolation, climatology is used for SST and the SSH measurement is assumed to have a zero deviation. This means that until the field deviates from the climatology, the extra data has added no value and MODAS reverts to climatology. 
Once the data is in a useful form, MODAS begins with the climatology profile and then correlates variations in the SSH and SST to variations in the subsurface temperature. The regression relationships used here were constructed by performing a least-squares regression analysis on archived temperature and salinity profiles. This is a three step process starting with the computation of regional empirical orthogonal functions from the historical temperature and salinity profiles. The second step is to express the profiles in terms of an empirical orthogonal function series expansion. The final step is to perform regression analysis on the profile amplitudes for each mode, truncating the series after three terms. This is possible because of the compactness of the empirical orthogonal function representation.

Once the subsurface temperatures have been revised, MODAS adjusts the subsurface salinity profile using the relationship between temperature and salinity. This new profile is referred to as a synthetic profile. Synthetic profiles only utilize these regression relationships down to a depth of $1500 \mathrm{~m}$ due to the decreasing reliability of the relationships at depth.

MODAS is also able to include measurements from in-situ CTDs and XBTS. The first guess field is the field generated by the dynamic climatology, and the in-situ profiles are subtracted from it to get residuals. Optimal interpolation is once again used to update the temperature field and from the temperature field the salinity field can be generated. This salinity field then serves as a first guess field for the inclusion of the salinity profiles (Fox, 2002).

\section{SATELLITE ALTIMETRY DATA ASSIMILATED INTO MODAS}

The Navy currently uses satellite altimeters to measure SSH that affect their operations. Of primary interest is mesoscale variability. Meandering fronts and eddies can significantly change the temperature and salinity structure of the ocean. This importance is clearly seen in sonar dependent operations such as ASW. Sonar range can be greatly helped or hindered by the acoustic environment created by the salinity, temperature, and density. Altimeters also provide the SSH and SST measurements that MODAS uses in its optimal interpolation.

While monitoring mesoscale variability is of prime importance to the Navy, an emerging secondary role for Navy altimeters is monitoring continental shelf and coastal zones. As the Navy conducts more and more operations in littoral waters, the ability to predict nearshore parameters will have increasing importance. Altimeter data can be used to get up-to-date information on rapidly changing near-shore characteristics such as tides and wave height. These are important issues for anyone dealing with mine detection, beach operations, or ship routing.

Altimeters have also been used to measure the flow through important straits, such as the Tsushima Strait, and to measure large-scale circulation. The first of these helps researchers and modelers to develop constraints on local numerical models. Large-scale circulation measurements can also help in the development of models by aiding in error correction. They also help explain the local environment that is often affected by not just local forcing, but large-scale circulation variations as well.

Satellite altimeters can provide a great variety of data, but no single altimeter can provide measurements on all desired time and length scales. Different parameters must be sampled at different frequencies if they are going to be of any use. For instance, sea surface height must be sampled every 48 hours while wave height must be sampled every three hours. While different ocean features all have different time and spatial scales, only the requirements for observation of mesoscale features are presented here as an example (Jacobs et. al., 1999 $[5])$.

In order for an altimeter to efficiently and accurately sample mesoscale features, there are several requirements placed on its accuracy, orbit, and repeat period. A satellite altimeter must produce measurements that are accurate to within $5 \mathrm{~cm}$, or the errors that propagate down into the temperature and salinity calculations will be unacceptable. With an error of only 5 $\mathrm{cm}$, the error in the temperature calculation can be $1-2^{\circ}$ C. Satellites should also have an exact repeat orbit to maximize the usefulness of the data collected. Without an exact repeat orbit the only way to get differences in sea surface heights is to use only the data from points where the satellite crosses the track of another altimeter or itself. An exact orbit is considered to be a $1 \mathrm{~km}$ wide swath of a predefined ground track. Finally, the period of a single satellite should be greater than the typical 20 day time scale of a mesoscale feature. If two satellites are used, then they should be spaced so that a point on the ground is not sampled more than once in a 20 day period (Jacobs et. al., 1999).

As described earlier, systems such as MODAS rely heavily on the information provided by these satellites. MODAS uses interpolation to estimate SSH at points that the satellite did not cover. If the ground track spacing is too coarse then the optimal interpolation scheme of MODAS will begin introducing errors into the fields between the tracks. It is important that the satellites be properly set up so that a maximum amount of information can be gathered with a minimum amount of error (Jacobs et. al., 1999). 


\section{NAVY's Weapon ACOUSTIC PRESet}

A Weapon Acoustic Preset Program (WAPP) is used to get automated, interactive means of generating $\mathrm{Mk} 48$ and Mk 48 ADCAP acoustic presets and visualizing torpedo performance. It combines the Mk 48 Acoustic Preset Program (M48APP) and the Mk 48 ADCAP Acoustic Preset Program (MAAPP) into a single integrated package. The Royal Australian Navy as a part of the Collins Class Augmentation System (CCAS) also uses the M48APP, and the Royal Canadian Navy has changed the M48APP for Java. The program is based around a graphical user interface that allows the user to enter the environmental, tactical, target, and weapon data. With these user specified parameters, the program then performs a series of computations to generate accurate acoustic performance predictions. The output includes a ranked list-set of search depth, pitch angle, $\mathrm{LD}$, and effectiveness values, an acoustic ray trace, and a signal excess map (NUWC, 2002 [6]).

The Environmental Data Entry Module (EDE) is a simple GUI that allows the user to enter a variety of environmental parameters (Fig. 1). The sea surface fields allow the user to specify wind speed, wave height, and sea state based on either the World Meteorological or Beaufort scale conventions. The three fields are coupled so that an entry into one field will bring up the appropriate default values for the others. The bottom condition field allows the user to specify the bottom depth and to choose the bottom type from a list of possibilities. The bottom of the GUI is devoted to the water column characteristics and a sound speed profile. The temperature, sound speed, and depth are all in the appropriate English units. The volume scattering strength (VSS) is in dB. The additional fields include the latitude, longitude, the profile name, and the table group identifiers (NUWC, 2002).

Once the environmental parameters have been entered then the user can move on to the Acoustic Module Preset Display. This GUI allows the user to specify a number of parameters about the weapon, the target, and the way the weapon should search (Fig. 2). The list-set on the right side of the GUI displays a series of search depths, pitch angles, laminar distances, and effectiveness values. The effectiveness values for the various presets are based on expected signal excess and ray trace computations. Both plots can viewed from a pull-down menu. These provide a visual representation of the acoustic performance of the Mk 48 (NUWC, 2002 [6]).

In addition to automatically computing the most effective preset combination for a given set of environmental parameters, the program also allows the user to manually examine the effectiveness of any allowable preset combination via the signal excess and ray trace plots. The program also allows the user to save the tactical preset list and the accompanying environmental data. The data is stored locale to the weapon module and can be recalled later or transferred via a network to the combat control system.

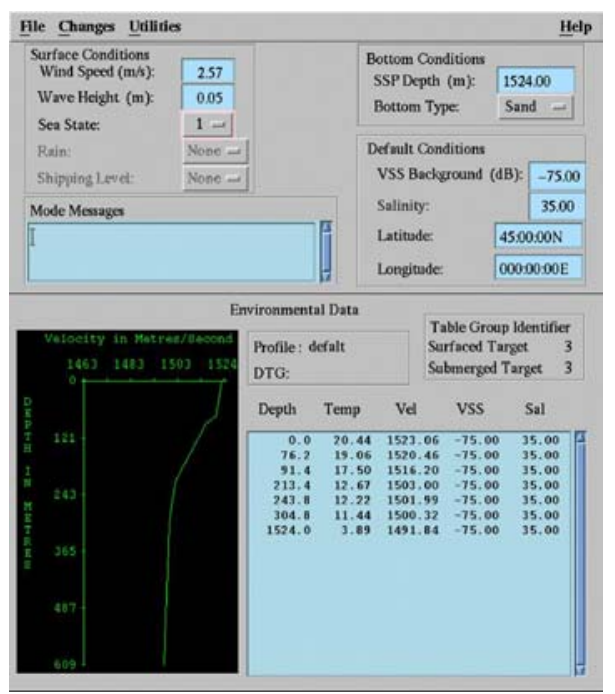

Fig. 1. EDE Interface.

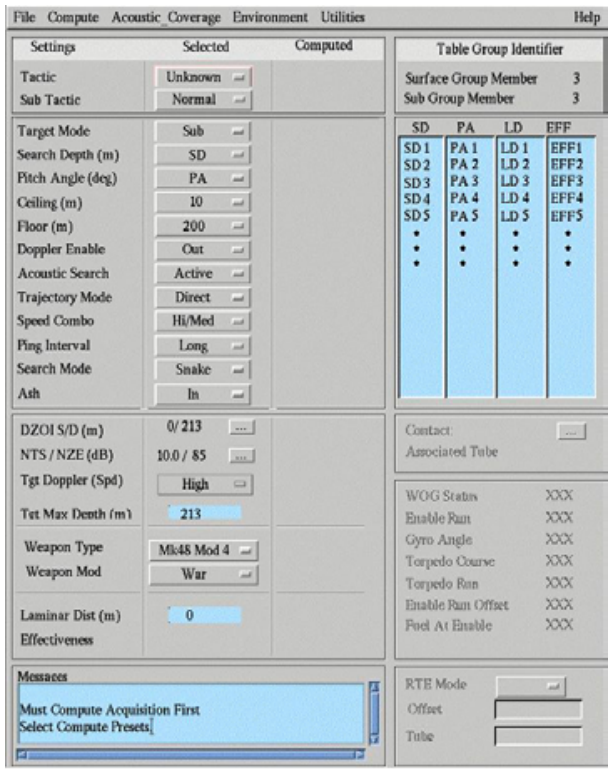

Fig. 2. Acoustic Preset Module Display

\section{Statistical Analysis}

\section{A. Input and Output Difference}

The difference of the two sets of input METOC data (GDEM and MODAS) $\psi_{\text {input }}$ or the two sets of output weapon preset data (running using GDEM and MODAS) $\psi_{\text {output }}$

$$
\Delta \psi(\mathbf{r}, t)=\psi_{M}(\mathbf{r}, t)-\psi_{G}(\mathbf{r}, t)
$$


represents the ocean data update using satellite and insitu observations (input) and the effect of using satellite and in-situ observations on the weapon preset (output). Here $\psi_{M}$ and $\psi_{G}$ are the variables (either input or output) using GDEM and MODAS, respectively. We may take the probability histograms of $\psi_{M}$ and $\psi_{G}$ to show the difference of the statistical characteristics.

\section{B. Root Mean Square Difference}

GDEM and MODAS have different grid spacing: $1 / 2^{\circ} \times 1 / 2^{\circ}$ in GDEM and $1 / 12^{\circ} \times 1 / 12^{\circ}$ in MODAS. For a GDEM cell, one data is available for GDEM and 36 data for MODAS. The root-mean-square difference (RMSD),

$$
\operatorname{RMSD}=\sqrt{\left[\frac{1}{N} \sum_{i=1}^{N}\left(\psi_{M}^{(i)}-\psi_{G}\right)^{2}\right]}
$$

is commonly used to represent the difference in the input and output data. Here, $N(=36)$ is the total MODAS data number in a GDEM cell. The RMSD can be computed for either the input data to the weapon preset model such as the temperature, salinity, or sound speed, or it can be computed for the output data such as nondimensional detection area.

\section{COMPARISON BETWEEN GDEM AND MODAS IN THE GULF STREAM REGION}

\section{A. Data}

In order to make a meaningful comparison of MODAS and GDEM data, a sufficiently large data set had to be obtained. The Area of Interest (AOI) also needed to be an area where the ocean environment fluctuated on a fairly short time scale. The GDEM data in March and MODAS data on March 15, 2001 was obtained for the area off the North American coast corresponding to $40^{\circ}$ $35^{\circ} \mathrm{N}$ latitude and $75^{\circ}-70^{\circ} \mathrm{W}$ longitude (Fig. 3).

Due to the differing resolutions of GDEM and MODAS, this area provided 117 GDEM profiles and 1633 MODAS profiles (Fig. 4). Each profile was simply a text file that consisted of a header row and columns of data. The header row contained the number of depths the profile covered, the file's name and the latitude and longitude of the profile.
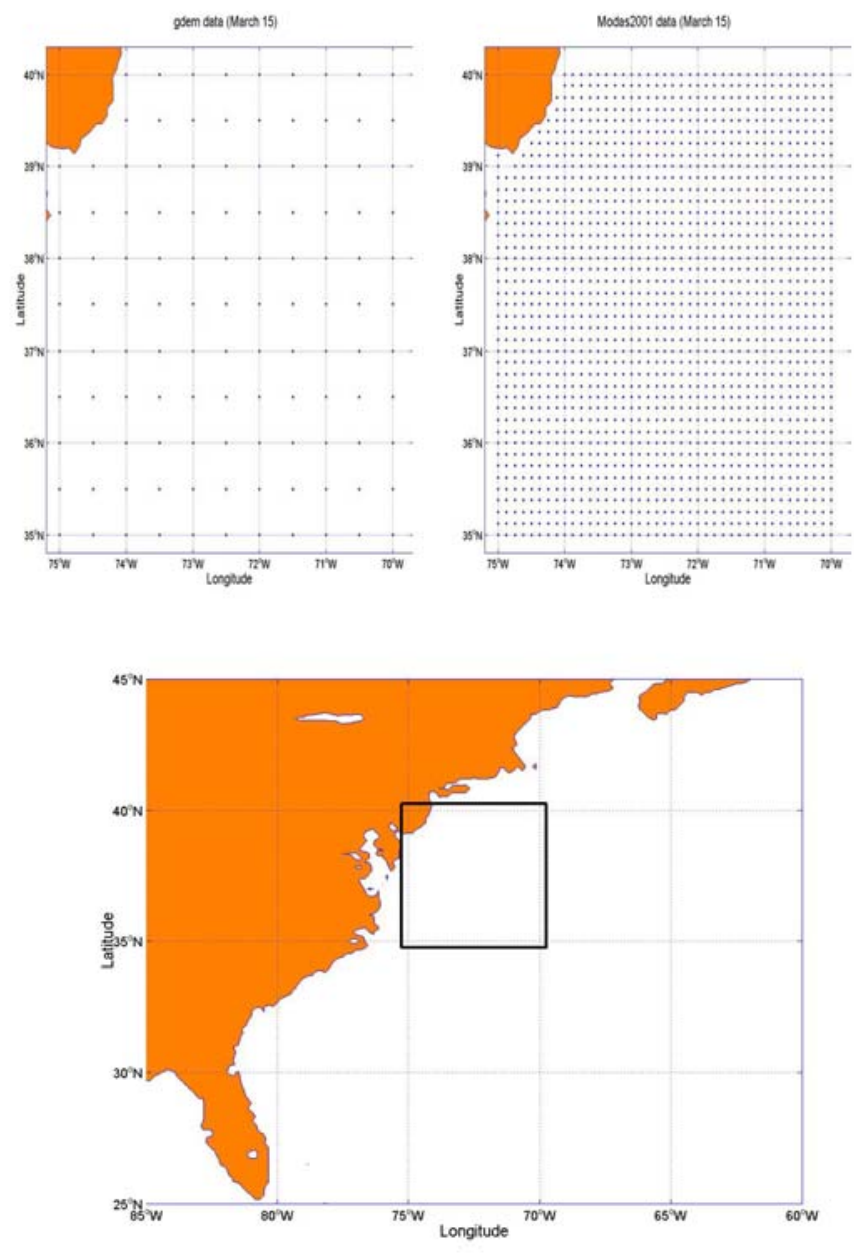

Fig. 3. Area of Interest

Fig. 4. GDEM and MODAS data points

The columns corresponded to depth in feet, the temperature in degrees Fahrenheit, the sound speed velocity in feet per second, a volume backscatter value, and salinity in PSU. Despite the common use of International units in scientific experiments it was necessary for the profiles to be set up in the appropriate English units. The Weapon Acoustic Preset Program 
(WAPP), the program used to generate the presets from the profiles, requires inputs to be in English units.

\section{B. Difference Between GDEM and MODAS}

While GDEM will often give background ocean environment at a given place, MODAS is known to provide more current and synoptic interpretations of the environment. The amount of accuracy MODAS adds is in proportion to the scale on which ocean parameters vary. For areas such as the Gulf Stream, where environmental factors are known to vary rapidly on a relatively small time scale, it is expected that there would be at least a few areas where the two data sets differ. It is these areas that are of particular interest since the difference in the weapon presets should be greatest.

On the surface, the GDEM data provided a view of the temperature distribution that consisted of smooth, uniformly spaced lines of constant temperature that were consistent with the overall flow of the region (Fig. 5). The cool water on the shelf gradually gives way to the warm water flowing north along the Gulf Stream. The GDEM generated surface salinity distribution is extremely similar to the surface temperature distribution and is consistent with the Gulf Stream region. Fresher water lies inland and the salinity increases with distance from the shore. The only variation is in the northeastern section where there is a slight intrusion of the salty offshore water.
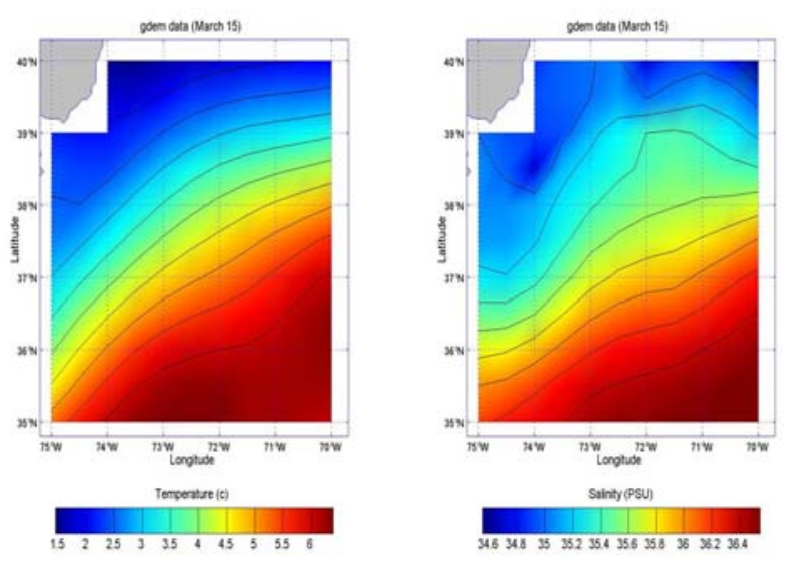

Fig. 5. March Surface Temperature and Salinity Distribution from GDEM.

As expected, the GDEM and MODAS distributions are, overall, fairly similar in both their range of values and overall distribution. They are similar to each other in shape, and both show areas of cool fresh water near the coast and areas of warm salty water lying offshore. There are, however, a few differences, with the intrusion of warm salty water in the northeastern section of the MODAS figure being the most notable. There is also an area of high temperature in the lower right corner of the MODAS figure that does not show up in the GDEM figure. In general the MODAS figure shows the water increasing in temperature and salinity much more rapidly as the distance from the coast increases (Fig. 6). The GDEM figure showed a gradual increase in temperature and salinity starting in the top left corner and continuing almost entirely down to the lower right corner. The MODAS figure shows the water reaching maximum temperature and salinity quickly and then staying constant to the lower right corner.
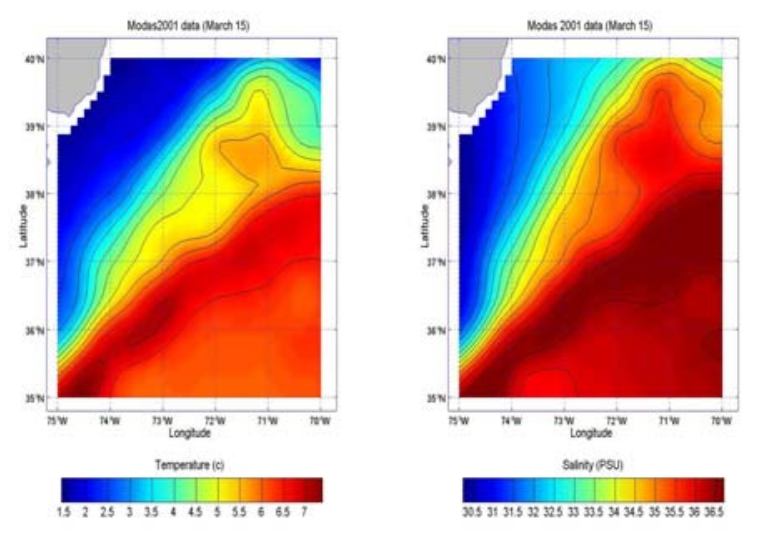

Fig. 6. MODAS Generated Surface Temperature and Salinity Distribution on March 15, 2001.

While the GDEM and MODAS data offer similar ranges of temperatures, salinities, and sound speeds at the surface, the distribution of the values is quite different. The histograms in Appendix A reveal that while the temperature values reported by both data sets are similar, the MODAS data has a higher proportion of profiles located in the $6^{\circ}-7^{\circ} \mathrm{C}$ range. The difference in the salinity graphs is even more drastic with the bulk of the GDEM values located in the middle of the range and the MODAS values split between the high and low ends of the range. The sound speed graph indicates that MODAS typically reported higher sound speeds than did the GDEM data. This is not too surprising since sound speed in the upper water column tends to be tied closely to temperature, and the MODAS data indicated warmer water than the GDEM data.

Increasing depth to $50 \mathrm{~m}$ and then $100 \mathrm{~m}$, it is clear to see that, for temperature, the distribution of the values over the range for both sets of data is quite similar. There is still a slight preference in the MODAS graphs towards higher temperatures, but it is not as drastic as was seen on the surface. Salinity is much the same, with the difference in shapes of the two figures more a factor of the small number of GDEM profiles as compared with the number of MODAS profiles. Sound speed is the only area where the two data sets continue to diverge. From the $50 \mathrm{~m}$ and $100 \mathrm{~m}$ sound speed figures it is clear that, with depth, the MODAS data indicates increasing sound 
speed and the GDEM data predicts some sort of sound speed minimum at depth. This is causing the peak on the MODAS graph and the peak on the GDEM graph to move away from each other as depth increases.

By $2000 \mathrm{~m}$ the temperature and salinity histograms for the two data sets are virtually identical. At this point any perceived difference in the two is solely a factor of the difference in the number of profiles between the two data sets. For the sound speed figures this is the point of maximum separation. The GDEM data indicates low sound speeds representative of a deep sound channel, whereas the MODAS data indicates that the sound speed has increased to this point. After this point the GDEM values begin rising again to match the MODAS data. While the distribution of the values over the range is a useful tool in examining the inputs, it is the difference between the inputs that is of real importance. The figures in Appendix B show the RMS difference of the inputs for a variety of depths. From the surface temperature figure in Appendix B, the RMS difference of temperature peaks out in the lower left corner of the AOI at about $2^{\circ}$ C. Besides the peak, the other significant area is the ridge starting in the lower left corner and running to the middle top of the figure. This corresponds to a narrow region where the GDEM distribution warmed slower than the MODAS distribution moving from the coast out to sea. The warm water intrusion is represented by the gradual increase in height of the ridge. The salinity difference at the surface is nearly zero for most of the AOI and reaches its maximum value of 4.5 PSU along the top of the region. The derived sound speed RMS difference, as expected, is smallest far from the coast where the difference in temperature and salinity is smallest and increases towards the coast.

As depth increases, the RMS difference in temperature and sound speed changes slowly, but the difference in salinity drops off quickly. Neither the temperature nor sound speed difference changed significantly, but by 100 meters the RMS difference for salinity has gone down to values of less than 8 PSU. From 100 meters down, the temperature difference begins to decrease slowly, and by 2000 meters the RMS difference for both temperature and salinity has dropped to negligible levels for most of the AOI. This is expected since MODAS reverts to climatology at depth. Except for the profiles in the northwestern corner of the AOI that did not run as deep as the other profiles farther from the coast, all the RMS difference vs depth profiles in were remarkably similar. All of the temperature differences showed either a gradual decrease in the difference down to about 1000 meters or a slight increase in the difference immediately followed by a gradual decrease in the difference down to 1000 meters. At about 1000 meters the temperature differences all rapidly dropped to near zero.
The sound speed profiles all show the difference increasing down to a maximum value of $60 \mathrm{~m} / \mathrm{s}$ at around 2000 meters. After that the RMS difference drops off, and approaches zero by 3000 meters. The cause of the maximum at 2000 meters is lack of a deep sound channel according to the MODAS data. The MODAS profiles almost all have the sound speed steadily increasing down to the maximum depth whereas climatology indicates a sound speed minimum at $2000 \mathrm{~m}$. While there is some variation in how quickly the salinity
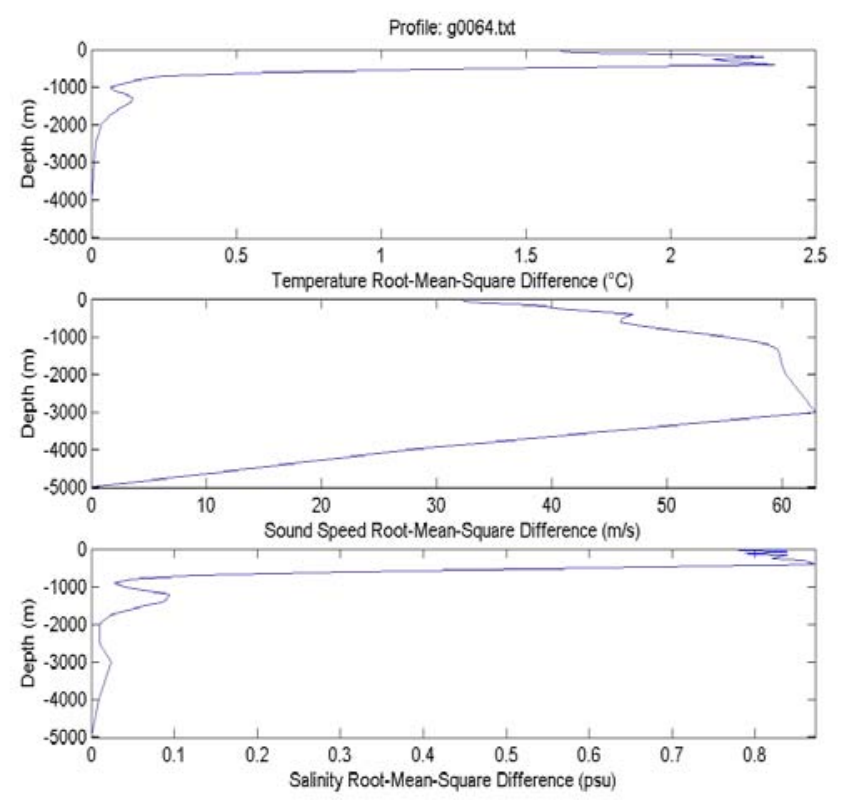

differences drop to near zero, they are less than 1 psu by 200 meters. Shown in Figure 7 is a representative RMS difference profile.

Fig. 7. RMS Differences of (a) temperature, (b) sound speed, and (c) salinity profiles.

\section{COMPARISON OF WEAPON ACOUSTIC PRESET USING GDEM AND MODAS}

The raw data was processed by the Naval Underwater Warfare Center (NUWC) Division Newport. They received the input profiles, ran them through the WAPP, and generated the output. Percentage coverage was calculated based on both surface (ASUW) and submarine (ASW) scenarios. The submarine scenario is a low Doppler scenario consistent with diesel submarine operations. The coverage percentages represent coverage in the target depth band, either shallow, mid, or deep. The coverage percentages were also normalized over acoustic modes to produce an output that was dimensionless. 


\section{A. Output Distributions}

The output provided by NUWC from the WAPP runs consisted of twelve different percentage coverage groups, three depth bands times two scenarios times the two different types of input data. For the non-SVP derived WAPP inputs, consistent values were used throughout the runs to ensure that any difference in the outputs was a result of differences in the GDEM and MODAS data. For each of the groups, basic statistics such as mean, maximum, minimum, and standard deviation were computed and then the data was constructed into histograms to give a visual representation of how the data was distributed.

In the shallow depth band ASUW scenario both MODAS and GDEM yielded mean coverage percentages that were very close to each other. While statistically the means are different, in real world applications a few percentage points difference is negligible (Fig. 8). From a users standpoint this means that both sets of data predicted about the same mean coverage for the AOI. The ASW scenario yielded similar results except for the fact that the two means were not even statistically different. While this seems to indicate that the two data sets are returning similar results, there are some important differences. First are the outliers on the GDEM graphs. Values in the high thirties to low fifties are extremely rare, yet the GDEM data indicate that in at least one location for the ASUW scenario and several for the ASW scenario, the weapon will perform to this level. The ASW scenario also had a rather significant number of GDEM profiles that generated below average coverage percentages. This would indicate that GDEM predicts that coverage will vary greatly with location. In comparison the MODAS values for both scenarios tended to be very consistent. Coverage percentage varies little with location due to the fact that most of the profiles lie within a very narrow range. Overall GDEM predicts excellent coverage some of the time and poor coverage the rest of the time. MODAS data on the other hand, indicates that coverage percentage will not be excellent anywhere but the expected values will be uniform over the whole shallow depth band region.
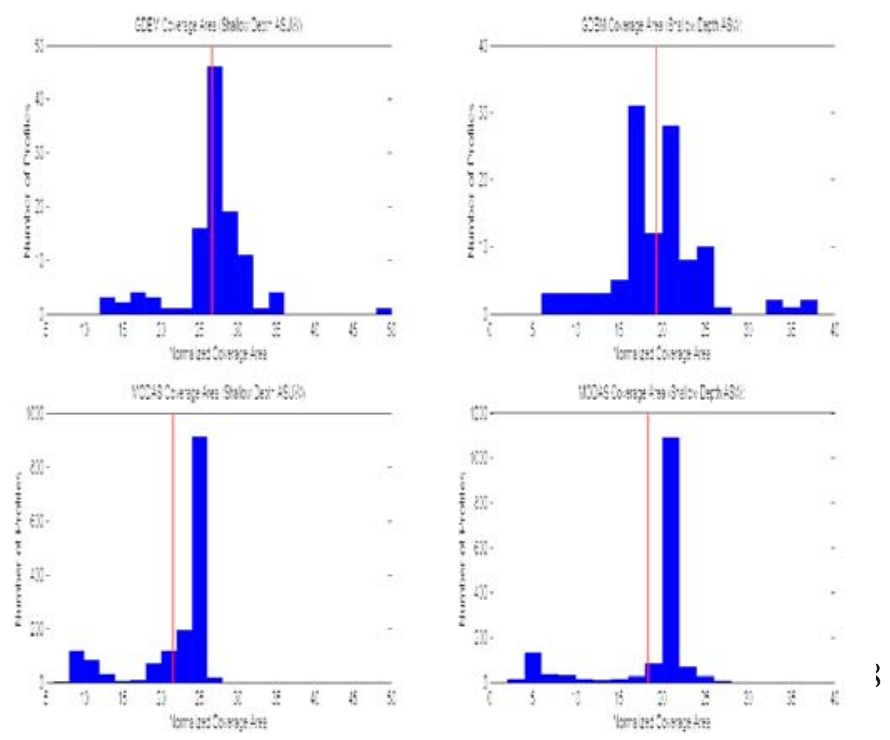

Fig. 8. Shallow depth band coverage percentage distributions: upper panels for GDEM and lower panels for MODAS, left panels for ASUW scenario and right panels for ASW scenario.

The mid depth band yielded results that were similar in distribution to the shallow depth band (Fig. 9). Across both scenarios the mean coverage of the GDEM data and the mean coverage of the MODAS data were statistically identical. Outliers were once again observed in the GDEM data, the larger outlier in the ASUW scenario, and the greater number of outliers in the ASW scenario. The wide dispersion of the GDEM derived coverage indicates that weapon effectiveness will vary depending on location. This is similar to the predictions for the shallow depth band and would indicate that GDEM predicts a water column that has varying coverage values depending on horizontal and vertical location. MODAS data once again indicates an overall performance in the region that is slightly less than the GDEM prediction; however, the MODAS data is grouped even more tightly than in the shallow depth band. The coverage in the ASW scenario in particular varies little about the mean value. This and the shallow depth band predictions indicate uniform coverage can be expected even at some depth.

In the deep depth band the graphs take on a slightly different shape, but they convey much the same meaning (Fig. 10). In both scenarios the GDEM graphs are weighted heavily to the right end, predicting that in the deep depth band coverage will be very good over most of the area. The ASUW scenario has the larger predicted values, but the values in the ASW scenario are still on the upper end of what is normal. The MODAS data predicts performance that is, while not particularly bad, still much more pessimistic than the GDEM predictions. 

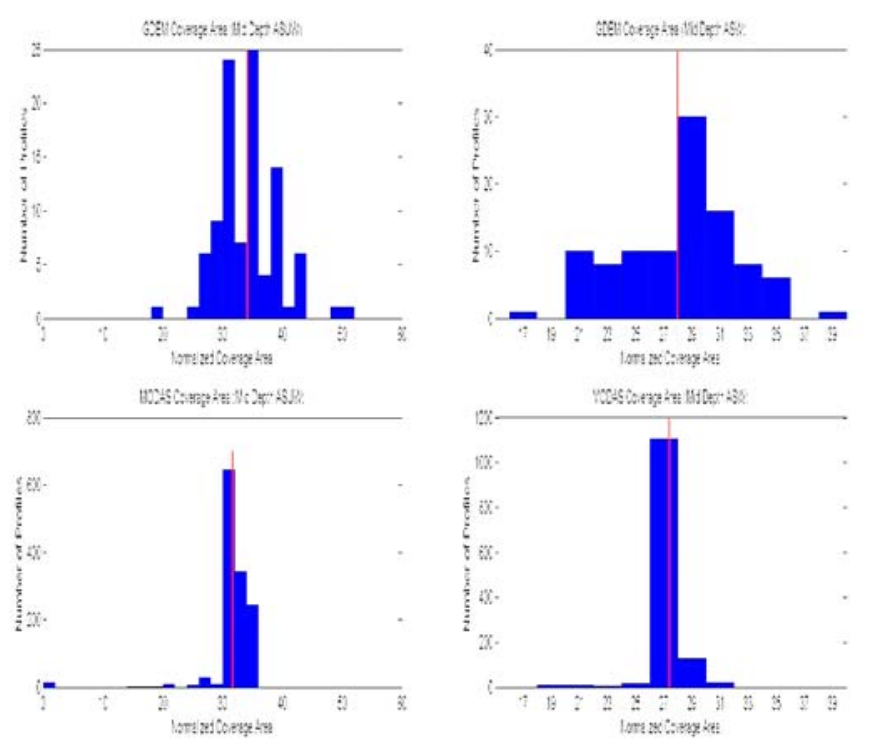

Fig. 9. Medium depth band coverage percentage distributions: upper panels for GDEM and lower panels for MODAS, left panels for ASUW scenario and right panels for ASW scenario.

For both scenarios the means of the GDEM and MODAS derived predictions are statistically different with the MODAS data providing the smaller mean in both scenarios. Although the dispersion of the GDEM data is large in both scenarios, the data is so heavily weighted towards the upper end that low GDEM coverage percentages are average values for the MODAS data coverage percentages. The MODAS data coverage percentages are once again tightly grouped; the uniformity of the predicted coverage percentages observed in the two other depth bands extends from the surface down to the selected maximum operating depth.
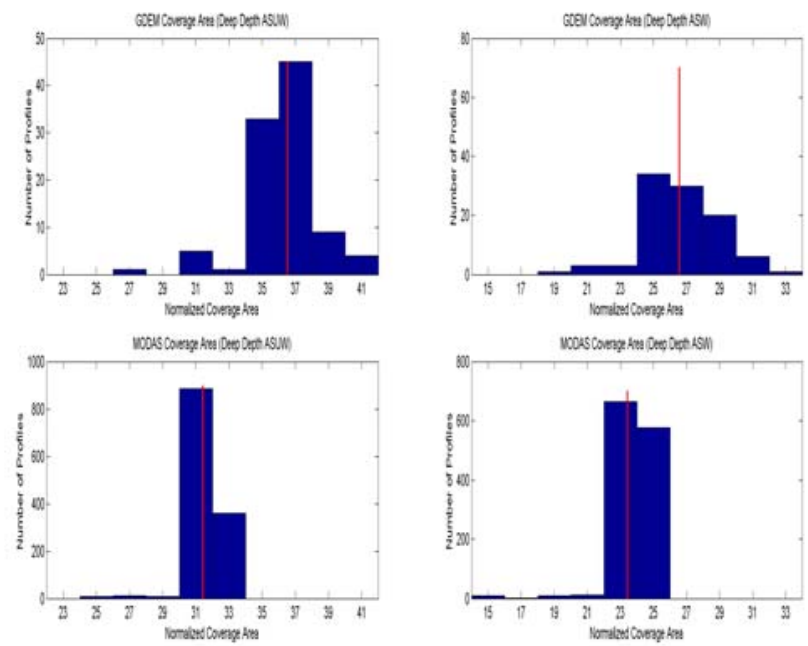

Fig. 10. Deep depth band coverage percentage distributions: upper panels for GDEM and lower panels for MODAS, left panels for ASUW scenario and right panels for ASW scenario.

\section{B. Difference of MK48 Acoustic Presets Using GDEM and MODAS}

For the shallow depth band, the RMSD in the percentage coverage area was small over most of the AOI, consistent with the similar means and range of values noted in the previous section (Fig. 11). The areas computed to have small RMSD coverage percentages also had small RMSD in temperature and salinity. In the region where the RMSD in temperature and salinity was largest, though, a large RMSD in percentage coverage is also observed. These larger values are likely areas where the GDEM data generated overly optimistic coverage percentage predictions. For the surface scenario, RMSDs of up to $25 \%$ are shown in the region around $39^{\circ} \mathrm{N} 73^{\circ}$ $\mathrm{W}$, and the warm salty intrusion observed on the MODAS data coincides with a second peak in the northeastern section of the graph. Overall the ASW scenario shows RMSDs that are similar to the ASUW scenario, the only difference being that the values are, on average, slightly smaller. The notable exception is the peak located at the top portion of the graph
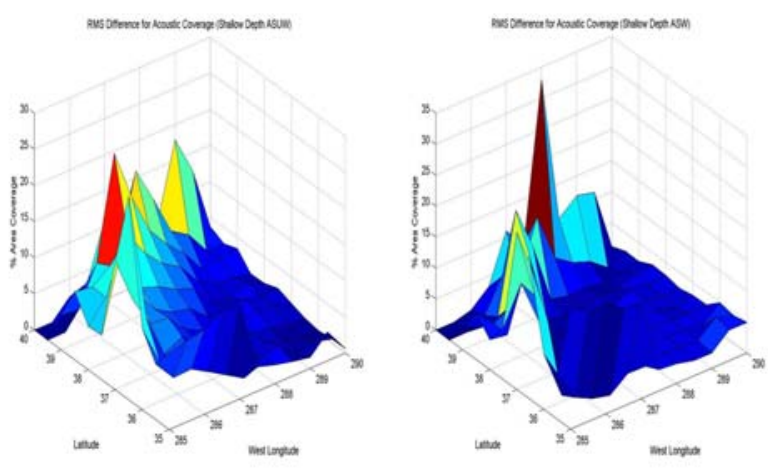

Fig. 11. RMSD for shallow depth band coverage: left panel for ASUW scenario and right panel for ASW scenario.

For the mid depth band the percentage coverage RMS difference for the ASUW scenario is simply a scaled down version of the shallow depth band ASUW graph (Fig. 12). This makes a great deal of sense considering the fact that the coverage percentage distributions for the shallow and mid depth ASUW scenarios were very similar. The real difference is in the ASW scenario. The single exceptional peak at the top of the previous graph is gone and the observed differences have become much smaller. Most of the RMS differences for the mid depth ASW scenario do not exceed $10 \%$. This is probably due to nearly identical coverage percentage means from both data sets, and the tighter grouping of the GDEM data 
coverage percentage predictions in the mid depth band ASW scenario. The RMSD values are small even in the areas where the temperature and salinity differences were observed to be large, such as in the upper section of the graph.
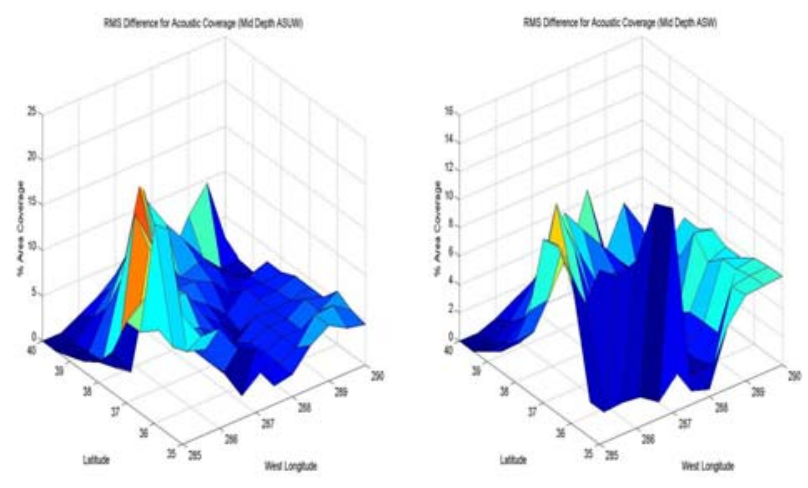

Fig. 12. RMSD for medium depth band coverage: left panel for ASUW scenario and right panel for ASW scenario.

The RMS differences observed in the deep depth band scenarios were smaller than those of the shallow depth band, but similar in magnitude to the mid depth band (Fig. 13). For the ASUW scenario the RMSD peaks near the northwestern corner of the AOI and then decreases steadily in steps heading toward the opposite corner. While the individual RMSD values seen are not as large as some of the ones in the other depth bands, more of the area has a non-negligible RMSD. The cause of this can be seen from the percentage coverage distribution for the deep depth ASUW scenario.

The GDEM data resulted in values that were almost all larger than the largest MODAS derived values. This overly optimistic prediction means that over a large portion of the AOI, the RMSD is going to be non-zero. The RMS difference in the ASW scenario changes very little from the mid depth band save for the fact that the values in the lower right corner are smaller. The coverage distributions for the deep ASW scenario were similar to the ASUW case, but the separation between the two means was not so pronounced. The result is a larger region where the RMSD is small or zero.

Both of these graphs match the pattern that has so far been observed in the other depth bands. The ASUW scenario has the higher RMS difference values, with areas of both high temperature and salinity differences corresponding to peaks on the graphs. The RMS difference values also approach zero moving toward the top left or bottom right corners. Also, as depth increases, the difference between the two data sets decreases causing the difference between the coverage percentages to decrease.
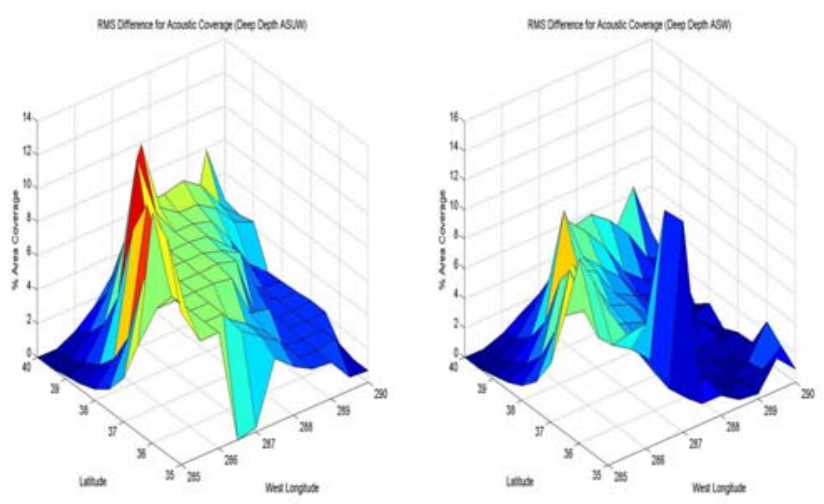

Fig. 13. RMSD for deep depth band coverage: left panel for ASUW scenario and right panel for ASW scenario.

\section{Conclusions}

\section{A. Discussion}

By looking at the RMSD in the temperature and salinity fields generated from the GDEM and MODAS data, it is possible to look for areas where the data differ significantly. It is at these points that the difference in the preset effectiveness should be the greatest. This was observed for both scenarios at all depth bands. The percentage coverage was the most different at points where both the temperature and salinity RMS difference was large. This was especially true for the shallow depth band where differences of $25 \%$ were observed for both scenarios. It is of interest to note that even at the surface the RMS differences for the temperature and salinity were never more than a few degrees or PSU. Even with only this slight increase in the accuracy of the inputs, a large increase in the accuracy of the prediction of the weapon effectiveness occurred. This seems to imply that the sensitivity of the presets to changes in the inputs is quite high.

From the output distributions it becomes clear that the GDEM derived coverage percentages indicate that weapon effectiveness should vary not only in the horizontal but also in the vertical. The implication is that in some areas coverage will be very high and in others the coverage will be very poor, but the tendency is for the coverage to be high for any given area. The MODAS derived percentages reveal that the exact opposite is true. The coverage will be consistent no matter what the horizontal location or depth band. This is an important 
result since prediction of weapon effectiveness is vital to mission planning and execution. In this case an unrealistic expectation in the weapons effectiveness would have resulted from the use of the GDEM data to predict the coverage percentages in the water column. The MODAS data also would have given the user the freedom to operate anywhere in the region knowing that their weapon would function about the same no matter the location.

\section{B. Future work}

The most obvious limitation of this work was the limited data set. Any future work should include data that covered a wider number of areas and times. Areas of strong thermal and salinity contrast are of particular interest. Various combinations of the user inputs into the WAPP should also be studied. The effects of variables such as bottom type and position (upslope/downslope) need to be addressed. Another avenue of study is the determination of how the number of altimeters affects the accuracy of the outputs. It has been determined that the presets are sensitive to the addition of satellite data. However, the effect of the number of satellite inputs still remains to be determined. Once this is done an optimal number of altimeters can be determined based on minimizing cost and maximizing preset accuracy.

\section{REFERENCES}

[1] Gottshall, Eric, Environmental Effects on Naval warfare simulations, MS Thesis in Physical Oceanography, Naval Postgraduate School, December 1997.

[2] Chu, P.C., E. Gottshall, and T.E. Halwachs, Environmental effects on Naval warfare simulations, Institute of Joint Warfare Analysis, Naval Postgraduate School, Technical Report, NPS-IJWA-98-006, pp.33, 1998.

[3] Teague, W.J., M.J. Carron, and P.J. Hogan, A Comparison Between the Generalized Digital Environmental Model and Levitus Climatologies. Journal of Geophysical Research, 95, 7167-7183, 1990.
[4] Fox, D.N., W.J Teague, and C.N Barron, The Modular Ocean Data Assimilation System (MODAS). Journal of Atmospheric and Oceanic Technology, 19, 240-252, 2002.

[5] Jacobs, G.A., C.N. Barron, M.R. Carnes, D.N. Fox, H.E. Hurlburt, P. Pistek, R.C. Rhodes, and W.J. Teague, Naval Research Laboratory Report NRL/FR/7320-999696, Navy Altimeter Data Requirements, 1999.

[6] Naval Undersea Warfare Center, Acoustic Presetting Status, Power Point view graphs, 2002. 
